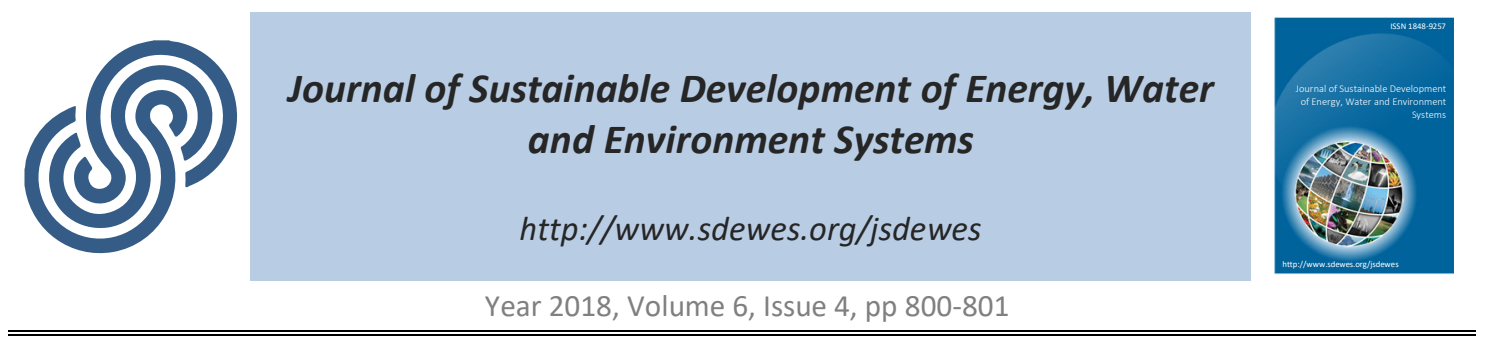

\title{
Journal of Sustainable Development of Energy, Water and Environment Systems - Volume VI
}

\author{
Neven Duić \\ Editor-in-Chief, Journal of Sustainable Development of Energy, Water and \\ Environment Systems
}

Cite as: Duić, N., Journal of Sustainable Development of Energy, Water and Environment Systems - Volume VI, J. sustain. dev. energy water environ. syst., 6(4), pp 800-801, 2018, DOI: https://doi.org/10.13044/2018.06.edt

The Journal of Sustainable Development of Energy, Water and Environment Systems (JSDEWES) is an international journal dedicated to the improvement and dissemination of knowledge on methods, policies and technologies for increasing the sustainability of development by de-coupling growth from natural resources and replacing them with knowledge based economy, taking into account its economic, environmental and social pillars, as well as methods for assessing and measuring sustainability of development, regarding energy, transport, water, environment and food production systems and their many combinations.

In total 48 manuscripts were published in Volume VI, all of them reviewed by at least two reviewers. The Journal of Sustainable Development of Energy, Water and Environment Systems would like to thank reviewers for their contribution to the quality of the published manuscripts.

1. Prof. Miroljub Adzic

2. Prof. Poul Alberg Østergaard

3. Prof. William H. Alfonso Piña

4. Dr. Amani Altmimi

5. Dr. Luis Alves

6. Prof. Hope Ashiabor

7. Prof. Lubka Atanasova

8. Prof. Milun Babic

9. Prof. Jan Baeyens

10. Dr. Tolga Baklacioglu

11. Prof. Jakov Baleta

12. Dr. Ipek Barut

13. Dr. Ilija Batas Bjelic

14. Prof. Marco Beccali

15. Prof. Vesna Borozan

16. Dr. Aleksandra Borsukiewicz

17. Prof. Sander M. Çalişal

18. Prof. Servio Tulio Cassini

19. Dr. Edgar Castillo

20. Prof. Ricardo Chacartegui

21. Prof. Pedro J. Coelho

22. Prof. Simona Colajanni

23. Prof. Silvio De Oliveira Junior
24. Prof. Matthias Dees

25. Prof. Nastia Degiuli

26. Prof. Umberto Desideri

27. Prof. Dimitar Dimitrov

28. Dr. Mansour Emtir

29. Dr. Mehmet Erdoğan

30. Mr. Bredo Erichsen

31. Prof. Bruno Fabiano

32. Prof. Veljko Filipan

33. Prof. Risto V. Filkoski

34. Prof. Gilles Fraisse

35. Dr. Alessandra Galatioto

36. Prof. Giuseppe Genon

37. Prof. Nesreen Ghaddar

38. Dr. Dionysios Giannakopoulos

39. Prof. Mario Giordano

40. Dr. Attila Göllei

41. Prof. Francesco Gonella

42. Dr. Veera Gnaneswar Gude

43. Prof. Petar Gvero

44. Prof. Dušan Gvozdenac

45. Dr. Eduard Hanslík

46. Prof. Kemal Hanjalic 
47. Prof. Pavel Hasal

48. Prof. Boshu $\mathrm{He}$

49. Prof. Patrick Hendrick

50. Prof. Carla Henriques

51. Prof. Ahmad Houri

52. Prof. Dagmar Juchelkova

53. Prof. Emmanuel Kakaras

54. Prof. Sanja Kalambura

55. Dr. Şiir Kilkiş

56. Prof. Jiří Jaromír Klemeš

57. Prof. Dardan Klimenta

58. Dr. Makoto Kobayashi

59. Dr. Iva Kovacic

60. Dr. Ivan Kozyatnyk

61. Prof. Svetislav Krstic

62. Dr. Jolita Kruopiene

63. Prof. Tarik Kupusovic

64. Prof. Igor Kuzle

65. Prof. Meng-Chieh, J. Lee

66. Dr. Tarzan Legović

67. Prof. Valerio Lo Brano

68. Dr. Ana M. López-Sabirón

69. Prof. Davor Ljubas

70. Prof. Marek Majdan

71. Prof. Anca Marina Marinov

72. Prof. Natasa Markovska

73. Dr. Fabio V. Matera

74. Dr. Brian Vad Mathiesen

75. Dr. Ken'Ichi Matsumoto

76. Prof. André Mesquita

77. Dr. Hrvoje Mikulcic

78. Prof. Kamal Mohammedi

79. Prof. Mousa Mohsen

80. Prof. Richard Moles

81. Dr. Harri Moora

82. Dr. Blanca Moreno

83. Prof. Claudia Morgado

84. Prof. Tatiana Morosuk

85. Dr. Karel Mulder

86. Prof. Boris. N. Kuznetsov

87. Prof. Endre Nagy

88. Prof. Sandro Nižetić

89. Mr. Tomislav Novosel

90. Prof. Eduardo Ortas

91. Dr. Hitesh Panchal

92. Prof. Rajkishore Patel

93. Prof. Patricia Pereira Da Silva

94. Dr. Stefan Petrovic

95. Prof. Antonio Piacentino

96. Prof. Matilde Pietrafesa

97. Prof. Nada Pop-Jordanova

98. Prof. Dr. Andrej Predin

99. Dr. Meysam Qadrdan

100.Dr. Arne M. Ragossnig
101.Prof. Nikola Rajakovic 102.Prof. Predrag Raskovic 103.Dr. Alberto Ríos 104.Prof. Gianfranco Rizzo 105.Dr. Alessandro Romagnoli 106.Prof. José Efren Ruelas Ruiz 107.Mr. Ilija Sazdovski 108.Prof. Daniel Rolph Schneider 109.Dr. Hans Schnitzer 110.Prof. Marek Ściążko 111.Dr. Vinod Kumar Sharma 112.Dr. Beatrice Smyth 113.Prof. Vladimir Soldo 114.Dr. Viola Somogyi 115.Dr. Maria - Loredana Soran 116.Dr. Antonio Soria-Verdugo 117.Prof. Svetlana Stevovic 118.Prof. Rodney Stewart 119.Dr. Mirko Stojiljkovic 120.Prof. Vladimir Strezov 121.Prof. Sanna Syri 122.Prof. Ireneusz Szczygiel 123.Prof. Zeljko Tomsic 124.Prof. Lamberto Tronchin 125.Dr. Koichi Unami 126.Prof. Krzysztof Urbaniec 127.Dr. Rudi Henri Van Els 128.Dr. Sharifah Rafidah Wan Alwi 129.Dr. Endong Wang 130.Dr. Douglas Wittmann 131.Prof. Sendren Sheng-Dong Xu 132.Dr. Cihan Yildırım 133.Mr. David Zambrana Vasquez 134.Prof. Aleksander Zidanšek 135.Dr. Katarzyna Zwarycz-Makles 136.Dr. Tea Žakula 137.Prof. Marija Živić 